\title{
More on Quantifiers in Comparative Clauses
}

\author{
Jon Gajewski \\ University of Connecticut
}

\section{Introduction}

The focus of this paper is the interpretation of DP quantifiers in clausal comparatives, cf. (1). Recent work has greatly advanced our understanding of this topic (Schwarzschild \& Wilkinson 2002, Schwarzschild 2004, Heim 2006a), yet several puzzles remain. In particular, though recent advances have rectified many of the problems encountered by earlier theories (von Stechow 1984, Rullmann 1995), it is unclear whether they retain all the benefits of these earlier theories. A case in point is the explanation of the unacceptability of downward entailing quantifiers in comparative clauses, illustrated in (2).

(1) Mary is taller than every boy is.

(2) *Mary is taller than no boy is.

One goal of this paper is to reconcile the advances made by Schwarzschild, Wilkinson and Heim with an account of the unacceptability of downward entailing quantifiers in comparative clauses. The analysis I suggest will be different in character from the accounts of von Stechow and Rullmann, which depend on the undefinedness of a maximality operator. Rather I suggest (following another idea sketched in von Stechow 1984) that comparative clauses containing downward entailing quantifiers yield trivial truth conditions and that it is this triviality that underlies their unacceptability.

Once such an analysis of the downward entailing cases is in place, we will see that problems arise for another class of quantifiers, namely, non-monotonic quantifiers, such as exactly two boys, between five and ten girls and an even number of tapirs. The analysis of the downward entailing cases yields truth conditions that are too weak for non-monotone quantifiers.

Mary is taller than exactly three boys are.

Focusing on the case of exactly quantifiers, I will show that the problems can be resolved if we recognize that non-monotonic quantifiers have a complex structure. In particular, we must follow Landman (1998) and Krifka (1999) in treating exactly two as having the same denotation as two but triggering the obligatory

Many thanks to G. Chierchia, D. Fox, I. Heim, R. Schwarzschild, Y. Sharvit and the audience at SALT 18 for feedback on this work. Thanks to S. Beck and F. Landman for sharing their unpublished work on this topic with me. Special thanks to E. Guerzoni for comments and encouragement. The remaining errors are mine. 
application of implicature-generating mechanisms. I will show that such an analysis of non-monotonic quantifiers, coupled with a plausible pragmatic principle constraining the level at which implicatures are introduced, yields the correct truth conditions for comparative clauses containing non-monotonic quantifiers.

In Section 2, I sketch a semantic theory (M-theory) for comparatives that reflects the important features of Schwarzschild's (2004) and Heim's (2006a) theories, as well as the rudiments of an alternative theory (E-theory) that I will ultimately endorse. The two theories are compared on the basis of their predictions for certain basic cases. Both are found wanting. In Section 3, I review the predictions of the standard theory (von Stechow 1984) concerning downward entailing quantifiers in comparative clauses. M-theory and E-theory are evaluated against the standard theory on this point - to the advantage of Etheory. In Section 4, I turn to the problem that non-monotic quantifiers pose for E-theory. The solution based on Landman (1998) and Krifka (1999) is sketched. Section 5 provides additional support for E-theory. Section 6 concludes.

\section{The Basic Problem}

In the course of the paper, I will compare two theories of the meaning of clausal comparatives. The first, which I will refer to as the maximality theory (MTheory), represents the insights of Schwarzschild \& Wilkinson (2002), Schwarzschild (2004) and Heim (2006a). The unique features of this approach are (i) that it places a scope-taking operator with in the than-clause and (ii) it applies the set of degrees abstracted from the than-clause to the maximal degree to which the main clause is true - hence the name 'maximality' theory. The scope-taking operator I have placed in the than-clause is negation (cf. Schwarzschild 2008). Henceforth, I will refer to the set of degrees contributed by the comparative clause containing negation as $\mathrm{CC}$, and the set of degrees contributed by the main clause MC.

\section{M(aximality)-Theory}

For $\mathrm{A}$ an individual, $\mathrm{P}$ a scalar predicate and $\mathrm{Q}$ a DP quantifier

$\mathbf{A}$ is $\mathbf{P}$-er than $\mathbf{Q}$ is is True iff

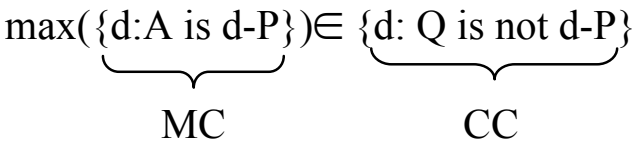

$$
\max (\mathrm{D}):=\mathrm{\imath d}\left[\mathrm{d} \in \mathrm{D} \& \forall \mathrm{d}^{\prime} \in \mathrm{D} \mathrm{d}^{\prime} \leq \mathrm{d}\right]
$$

This theory will be contrasted with an alternative theory, which I refer to as the existential theory (E-theory). I argue that it is the E-theory that is best able to capture the truth conditions of comparatives containing quantifiers. The Etheory incorporates the scope-taking element in the than-clause that constitutes a central part of the M-theory. E-theory, however, imposes a different relationship on $\mathrm{MC}$ and $\mathrm{CC}$. Whereas M-theory's truth conditions require the maximal 
member of $\mathrm{MC}$ to be a member of CC, E-theory's truth conditions merely require that $\mathrm{MC}$ and $\mathrm{CC}$ overlap, i.e. share some member. Hence the name 'existential' theory. See the Appendix for a fully spelled out LF.

(6) E(xistential)-Theory

For $\mathrm{A}$ an individual, $\mathrm{P}$ a scalar predicate and $\mathrm{Q}$ a DP quantifier

$\mathrm{A}$ is $\mathrm{P}$-er than $\mathrm{Q}$ is is True iff

$\{\mathrm{d}: \mathrm{A}$ is $\mathrm{d}-\mathrm{P}\} \cap\{\mathrm{d}$ : $\mathrm{Q}$ is not $\mathrm{d}-\mathrm{P}\} \neq \varnothing$

I assume throughout that gradable adjectives are monotone.

$$
\begin{aligned}
& \text { a. a function of type }<\mathrm{d},<\mathrm{e}, \mathrm{t}>>\text { is monotone iff } \\
& \forall \mathrm{x} \forall \mathrm{d} \forall \mathrm{d}^{\prime}\left[\mathrm{f}(\mathrm{d})(\mathrm{x})=1 \& \mathrm{~d}^{\prime}<\mathrm{d} \rightarrow \mathrm{f}\left(\mathrm{d}^{\prime}\right)(\mathrm{x})=1\right]
\end{aligned}
$$

b. $\quad \llbracket \operatorname{tall} \rrbracket=\lambda \mathrm{d} \cdot \lambda \mathrm{x} \cdot$ height $(\mathrm{x}) \geq \mathrm{d}$

We will now compare the basic predictions of M-theory and E-theory. The intuitions behind the two theories are clear when we consider cases comparing the measures of individuals. Consider the sentence (8). Under MTheory, to see if the sentence is true you find Mary's height and see if it is a standard of height that Bill fails to meet. Under the E-theory, you need only see if there is some standard of height that Mary meets and Bill does not. In this case, the conditions are equivalent. In more complex cases, the theories diverge in their predictions.

(8) Mary is taller than Bill is is True iff

a. E-theory: $\{\mathrm{d}$ : Mary is $\mathrm{d}$-tall $\} \cap\{\mathrm{d}$ : Bill is not $\mathrm{d}$-tall $\} \neq \varnothing$

b. M-theory: Mary's height $\in\{$ d: Bill is not d-tall $\}$

\subsection{Basic Facts about Quantifiers in CC}

In this section we see how M-theory and E-theory differ when we replace the embedded subject in a sentence like (8) with a true quantifier. We will approach the quantifiers by dividing them up in terms of monotonicity. As we shall see in more detail in Section 3, when Q in the schemata in (5) and (6) is upwardentailing, M-theory and E-theory give equivalent - and correct - truth conditions. For example, both theories predict (9b) to be true if and only if Mary's height is greater than the height of the shortest boy.

a. $Q$ is upward-entailing (UE) iff for all sets $A, B$ s.t. $A \subseteq B, Q(A) \Rightarrow Q(B)$

b. Mary is taller than some boy is.

E-theory: $\{\mathrm{d}$ : Mary is $\mathrm{d}$-tall $\} \cap\{\mathrm{d}$ :some boy is not $\mathrm{d}$-tall $\} \neq \varnothing$

M-theory: Mary's height $\in\{\mathrm{d}$ : some boy is not d-tall $\}$

The theories part ways first on the case of downward entailing quantifiers. As observed above, such quantifiers lead to unacceptability in comparatives. M- 
theory offers no semantic explanation for this unacceptability; it predicts that these cases have coherent, contingent truth conditions (though the matter is more complicated, as we will see in Section 3). For example, M-theory predicts that (10b) is true if and only if Mary is at most as tall as the shortest boy.

a. $\mathrm{Q}$ is downward entailing $(\mathrm{DE})$ iff for all sets $\mathrm{A}, \mathrm{B}$ s.t. $\mathrm{A} \subseteq \mathrm{B}, \mathrm{Q}(\mathrm{B}) \Rightarrow \mathrm{Q}(\mathrm{A})$ b. *Mary is taller than no boy is.

E-theory: $\{\mathrm{d}$ : Mary is $\mathrm{d}$-tall $\} \cap\{\mathrm{d}$ : no boy is not $\mathrm{d}$-tall $\} \neq \varnothing \quad \checkmark$ [tautology]

M-theory: Mary's height $\in\{\mathrm{d}$ : no boy is not d-tall $\}$ [wrong]

E-theory, on the other hand, offers a possible explanation for the unacceptability of DE-Qs in CC. Consider the truth conditions that E-theory predicts for (10b). They require that there be some standard of height that Mary meets and that no boy doesn't meet - that is, that every boy meets. Notice that there is always some standard the Mary and every boy meet. So, E-theory predicts the sentence to be a tautology. In Section 3, I will show that this is systematically the case with DEQs. I furthermore suggest that it is this systematic triviality that underlies the unacceptability of DE-Qs in comparatives. It appears that E-theory has an advantage over M-theory on DE-Qs.

Finally, we come to the case of non-monotonic quantifiers. Here we will see that M-theory has the advantage. M-theory correctly predicts that in order for (11b) to be true Mary both must be taller than two boys and cannot be taller than three boys. In other words, M-theory is able to impose a maximum on the height of the subject; in this case, the height of the third shortest boy, if there is one.

a. $\mathrm{Q}$ is non-monotonic (NM) iff $\mathrm{Q}$ is neither $\mathrm{UE}$ nor $\mathrm{DE}$

b. Mary is taller than exactly 2 boys are.

E-theory: $\{\mathrm{d}$ :Mary is d-tall $\} \cap\{\mathrm{d}$ :exactly 2 boys are not $\mathrm{d}$-tall $\} \neq \varnothing \quad$ [weak]

M-theory:Mary's height $\in\{\mathrm{d}$ : exactly 2 is not d-tall $\}$

E-theory, on the other hand, is incapable of imposing a maximum on the height of the subject. It can only impose a lower bound. Hence, E-theory predicts that (11b) is true if and only if Mary is taller than the second shortest boy. These truth-conditions are obviously too weak. In Section 3, I will show that E-theory can be fixed on this point once we pay more attention to the structure and interpretation of non-monotonic quantifiers.

\subsection{Summary}

In this section we have laid out two theories (M-theory and E-theory) of the interpretation of clausal comparatives. Though superficially similar, the theories yield divergent predictions about the truth conditions of comparative clauses containing DP quantifiers. M-theory and E-theory give equivalent results when the quantifier is UE. E-theory provides a foundation for a semantic explanation of the unacceptability of DE-Qs in comparatives; M-theory does not. Finally, Mtheory gives the right results for non-monotonic quantifiers, whereas E-theory 
yields truth conditions that are too weak. In Section 3, we examine the DE case in greater detail.

\section{Downward Entailing Quantifiers in Comparative Clauses}

In this section, we examine the predictions of M-theory and E-theory concerning DE-Qs against the backdrop of the predictions of the standard theory (von Stechow 1984, Rullmann 1995, Heim 2000). First, in Section 3.1, we review the compelling account of the unacceptability of DE-Qs in CC offered by von Stechow (1984). Then, in Section 3.2, we see that M-theory systematically fails to account for this unacceptability. Instead, M-theory predicts that a $\mathrm{CC}$ containing a DE-Q imposes a maximum on the measure of the subject. This will be significant in our discussion of non-monotonic quantifiers in Section 4. Finally, in Section 3.3, we see that E-theory predicts trivial truth conditions in these cases. I argue, following a suggestion in von Stechow (1984), that this triviality is responsible for the unacceptability of DE-Qs in CC.

\subsection{The Standard Theory}

As observed above, DE-quantifiers are (in general) unacceptable in comparative clauses. Consider the sentences in (12).
a. *Mary is taller than no boys are.
b. *Mary is taller than few boys are.
c. *Mary is taller than fewer than eight boys are.
d. *Mary is taller than not every boy is.
e. 'Mary is taller than at most three boys are.

The systematic exclusion of DE-Qs suggests that this is a semantically motivated phenomenon. Von Stechow (1984) and Rullmann (1995) offer an elegant semantic explanation for the restriction. They propose that the than-clause of a comparative denotes the maximum of a set of degrees, see (13). In what follows, I use Heim's (2000) implementation of this idea, in assuming that degree predicates are monotone functions of type $<\mathrm{d},<\mathrm{e}, \mathrm{t}>>$. ${ }^{1}$

Bill is taller than Fred is

LF: [-er [ $\mathrm{wh}_{1}$ Fred is $\mathrm{t}_{1, \mathrm{~d}}$ tall] $]_{2, \mathrm{~d}}$ Bill is $\mathrm{t}_{2, \mathrm{~d}}$ tall

TC: $\max (\lambda \mathrm{d}$.Bill is d-tall $)>\max (\lambda \mathrm{d}$.Fred is d-tall $)$

This theory provides an immediate explanation of the unacceptability of DE-Qs in comparative clauses.

\footnotetext{
${ }^{1}$ Von Stechow 84 and Rullmann 95 use an 'exactly' interpretation of degree predicates, but the need to scope out every (see (15) below) is the same.
} 
(14) *Bill is taller than no student is

LF: [-er [wh ${ }_{1}$ no student is $\mathrm{t}_{1, \mathrm{~d}}$ tall] $]_{2, \mathrm{~d}}$ Bill is $\mathrm{t}_{2, \mathrm{~d}}$ tall

TC: $\max (\lambda$ d.Bill is d-tall $)>\max (\lambda d$.no student is d-tall $)$

The set of degrees [ $\lambda$ d.no student is d-tall] has no maximum; every degree above the head of the tallest student is a standard of height that no student meets. Consequently, the application of a maximality operator to this set results in a presupposition failure. Hence the sentence is unusable. This is a great advantage for the standard approach, but it leads directly to its greatest failing. This approach yields incorrect truth conditions for many other quantifiers, see (15). To get the correct truth conditions in these other cases, the quantifiers must scope out, as in (16).

a. Bill is taller than every girl is. $\max (\lambda d$.Bill is d-tall $)>\max (\lambda d$.every girl is d-tall $) \quad$ [wrong!] the height of the shortest girl!

b. Bill is taller than most girls are. $\max (\lambda d$.Bill is d-tall $)>\max (\lambda d \text {.most girls are d-tall })^{2} \quad$ [wrong!]

(16) a. every $\operatorname{girl}_{\mathrm{x}}$ Bill is taller than $\mathrm{x}$ is.

b. most girls $\mathrm{x}_{\mathrm{x}}$ Bill is taller than $\mathrm{x}$ is.

The standard theory also fails with conjunctions like Bill and Fred, and nonmonotone quantifiers like exactly 2 girls. All need to be scoped out for the standard theory to derive the correct truth conditions. If these quantifiers are allowed to scope out, however, one needs a principled reason to block no student from scoping out, giving the coherent (17) as LF for (14):

no student $\mathrm{x}_{\mathrm{x}}$ Bill is taller than $\mathrm{x}$ is.

These faulty predictions are what M-theory was developed to correct. As we shall see, however, it is unclear how to maintain an account of the ban on DE-Qs under M-theory.

It must be noted that von Stechow 84 and Rullmann 95 argue that DE-Qs are not the only Qs that scope under max. They claim that NPIs, such as any, and disjunction also scope under max. [For M- and E-theories, scoping under the negation in $\mathrm{CC}$ is the equivalent of scoping under $\max$.]

Mary is taller than any boy is

$\max (\lambda$ d.Mary is d-tall $)>\max (\lambda d$.some boy is $\mathrm{d}$-tall $)$

\footnotetext{
${ }^{2}$ If there are an even number $\mathbf{n}$ of girls, then $\max (\lambda$ d.most girls are $d$-tall $)$ is the height of the $\mathbf{n} / 2$ th girl. For example, if there are six girls, the standard theory predicts that Bill only needs to be taller than three for the sentence to be true. Intuitively, though, he needs to be taller than at least four.
} 
Mary is taller than Bill or Fred is.

$\max (\lambda d$.Mary is d-tall $)>\max (\lambda d$.Bill or Fred is d-tall $)$

It has long been debated, without resolution, whether these are true occurrences of NPI any/narrow scope disjunction in English, or examples of free-choice any/disjunction (see Hoeksema 1983, von Stechow 1984b, Heim 2006a a.o.). There is evidence that free choice any can occur in comparatives: any in CC can be modified by absolutely/almost, like free choice any, unlike NPI any.

Mary is taller than almost/absolutely any other girl is.

Hoeksema (1983) presents evidence from Dutch suggesting that clausal comparatives do license NPIs. He shows that ook maar, a Dutch NPI that does not have free choice readings, may occur in clausal comparatives. If these are true NPIs in English, it may be that in these cases negation moves specifically to license the NPI (see a similar suggestion in Heim 2006a).

I will leave the issue of possible NPI DP quantifiers scoping under $\max$ /negation. In English at least, the evidence is inconclusive. Instead I will endorse a version of the Heim/Kennedy generalization concerning DP quantifiers and degree operators.

(21) DP quantifiers scope over max/negation in the than-clause. ${ }^{3}$

cp.Kennedy 1999/Heim 2000

\subsection{M-Theory on DE-Qs in $C C$}

M-Theory is designed to give a better account of Qs in CC. Under M-Theory there is no need to scope every boy, most boys, Bill and Fred, and exactly 2 girls out of the than-clause (cf. Schwarzschild \& Wilkinson 2002, Schwarzschild 2004, Heim 2006a). The correct reading is derived by these quantifiers scoping over negation within $\mathrm{CC}$.

(22) Mary is taller than every boy is.

M-theory: Mary's height $\in\{\mathrm{d}$ : every boy is not d-tall $\}$

Despite this success with previously problematic cases of UE-Qs, M-theory fails with DE-Qs. ${ }^{4}$ As observed before, M-theory predicts coherent, contingent truth conditions in these cases, providing no grounds for a semantic explanation of their unacceptability.

\footnotetext{
${ }^{3}$ This is just a quick and dirty version for present purposes, see the Appendix for the official version.

${ }^{4}$ Schwarzschild (2004) \& Heim (2006a) use a flexible scope max in CC, the point-to-interval (PI) operator. If that max scoped over a DE-Q in CC, there would be a presupposition failure. The question of where max scopes is the same as the scope issue for von Stechow (1984).
} 
(23) a. *Bill is taller than no girls are.

b. M-theory: Bill's height $\in\{\mathrm{d}$ : no girls are not d-tall $\}$

"Bill is at most as tall as the shortest girl"

This is not a particular failure with no girl but extends to all DE-Qs. The reason is that when Q is DE, CC is downward closed.

If $\mathrm{Q}$ is $\mathrm{DE}, \mathrm{CC}$ is $\mathrm{DC}$.

(25) A set of degrees D is downward closed (DC) iff $\forall \mathrm{d}, \mathrm{d}^{\prime}\left[\mathrm{d} \in \mathrm{D}\right.$ and $\left.\mathrm{d}^{\prime}<\mathrm{d} \rightarrow \mathrm{d}^{\prime} \in \mathrm{D}\right]$

Now recall that the truth conditions M-theory ascribes to a comparative sentence require the measure of the subject to be a member of CC. So when $\mathrm{CC}$ is downward closed, $\mathrm{M}$-theory predicts that $\mathrm{CC}$ imposes a maximum on the measure of the subject. In (23) the maximum is the height of the shortest girl; in (26), the maximum is the height of the tallest girl.

(26) *Bill is taller than not every girl is.

M-Theory: Bill's height $\in\{\mathrm{d}$ : not every girl is not $\mathrm{d}$-tall $\}$

"Bill is at most as tall as the tallest girl"

It is important to observe this consequence of M-theory, since this failure with DE-Qs is tied to directly to its success with NM-Qs.

\subsection{E-theory on $D E-Q s$ in $C C$}

E-theory inherits M-theory's success with quantifiers but also offers an account of DE-Qs. E-theory predicts that a CC containing a DE-Q yields trivial truth conditions (see von Stechow 1984: 34). It has been suggested that systematic triviality can be a cause of unacceptability (Barwise \& Cooper 1982, Chierchia 1984, von Fintel 1993). I will endorse such an analysis of the unacceptability of DE-Qs in CC under E-theory.

E-Theory inherits M-theory's advantages with UE-Qs. In fact, E-theory and M-theory are equivalent when Q in $\mathrm{CC}$ is UE. When the Q in $\mathrm{CC}$ is UE, CC is upward closed (UC). If a set of degrees D is upward closed, D contains the maximum of another set of degrees D' if and only if D overlaps D'.

(27) MC is DC and MC has a maximum.

(28) If Q is UE, CC is UC.

(29) A set of degrees D is upward closed (UC) iff

$\forall \mathrm{d}, \mathrm{d}^{\prime}\left[\mathrm{d} \in \mathrm{D}\right.$ and $\left.\mathrm{d}^{\prime}<\mathrm{d} \rightarrow \mathrm{d}^{\prime} \in \mathrm{D}\right]$

(30) For sets of degrees D, D' where D is DC and has a maximum and $\mathrm{D}^{\prime}$ is UC, $\max (\mathrm{D}) \in \mathrm{D}^{\prime}$ iff $\mathrm{D} \cap \mathrm{D}^{\prime} \neq \varnothing$ 
Hence, every boy, most boys and Fred and Bill are also handled in E-theory without scoping these Qs out. Now I show that E-theory predicts that whenever $\mathrm{Q}$ is DE the comparative sentence is trivially true.

This prediction is pointed to already in von Stechow's (1984:34) discussion of how Seuren (1973) might handle (31). ${ }^{5}$ Von Stechow observes that (31) is a tautology.
a. *Sue is smarter than neither Bill nor Mary is
b. ( $\exists d)$ [Sue is d-smart \& (Bill or Mary is d-smart)]

As observed in (27), MC is always DC and, when CC contains a DE-Q, CC is DC as well. These two observations nearly, but do not quite, guarantee that when $\mathrm{Q}$ is DE a comparative sentence is always true, under E-Theory. Recall that E-theory requires that the intersection of $\mathrm{MC}$ and $\mathrm{CC}$ is not empty. When $\mathrm{MC}$ and $\mathrm{CC}$ are both downward closed, it means that they are both initial segments of the scale when non-empty. Two non-empty initial segments of a scale must overlap.

It is plausible to assume that MC is always non-empty. The lexical entry for a gradable adjective properly contains a presupposition that its individual argument has a measure on the relevant scale. ${ }^{6}$

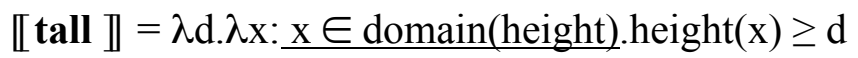

I will stipulate that $\mathrm{CC}$ is presupposed to be non-empty, as well. ${ }^{7}$ This is necessary, for example, to rule out sentences such as (33). If there were no students, CC, given in (33), would be empty and (33) false in that case. In many cases, the presupposition that $\mathrm{CC}$ is non-empty is indistinguishable from the presupposition that the restrictor of $\mathrm{Q}$ is non-empty.

a. *Mary is taller than not every student is tall.

b. $\{$ d: not every student is not $\mathrm{d}$-tall $\}$ ( $=\{\mathrm{d}$ : some student is $\mathrm{d}$-tall $\})$

Assumption: $\mathrm{CC}$ is presupposed to be non-empty.

Given (24), (32), and (34), it follows that when Q is DE and the comparative clause is defined, it will always be true. I propose that it is this systematic triviality explains the unacceptability of (36).

(35) The intersection of any two non-empty DC sets of degrees is non-empty.

$$
\text { *Fred is taller than no student is }
$$

\footnotetext{
${ }^{5}$ Note that Seuren (1973) differs from E-theory in scope of negation; but this is irrelevant to the triviality account.

${ }^{6}$ On certain closed scales, the meaure assigned to an individual might be $\mathbf{0}$.

${ }^{7}$ This issue doesn't arise in von Stechow's example (31) since CC contains referring expressions.
} 
E-theory: $\{\mathrm{d}$ : Fred is $\mathrm{d}$-tall $\} \cap\{\mathrm{d}$ : no student is not $\mathrm{d}$-tall $\} \neq \varnothing$

For explicit proposals that certain kinds of tautologies and contradictions should be considered ill-formed see Chierchia (1984), Gajewski (2002) and Fox and Hackl (2006).

\subsection{Summary}

In this section, we have reviewed the standard account of the unacceptability of DE-Qs in CC due to von Stechow (1984). We observed that the standard accounts correct predictions in this case lead to wrong predictions for many other quantifiers. M-theory solves many of the problems of the standard account, but fails to offer a principled account of DE-Qs. Instead it predicts that DE-Qs in CC impose a maximum on the measure of the subject.

I have argued that E-theory fares the best so far. It yields equivalent results to the M-theory in the case of UE-Qs in CC. By contrast, under the assumption that $\mathrm{CC}$ is non-empty, E-Theory predicts that when $\mathrm{Q}$ is $\mathrm{DE}$ a comparative sentence has trivial truth conditions. I have proposed that triviality can explain the unacceptability of DE-Qs in this case. So, E-theory provides plausible analyses of both UE and DE-Qs in CC, unlike M-theory and the standard theory. As we see in Section 4, however, M-theory is superior to Etheory in its predictions concerning NM-Qs in CC. I will show, however, that Etheory can be amended to match M-theory's predictions.

\section{Non-monotonic Quantifiers (NM-Qs)}

The case of NM-Qs provides crucial motivation for M-theory (cf. Heim 2006a, Schwarzschild 2008). M-theory gets NM-Qs like exactly two NP and 10 to $15 \mathrm{NP}$ just right; E-theory predicts truth conditions that are too weak.

Mary is taller than exactly two boys are.

\#E-theory: $\{\mathrm{d}$ :Mary is d-tall $\} \cap\{\mathrm{d}$ :exactly 2 boys are not $\mathrm{d}$-tall $\} \neq \varnothing$ [weak]

"Mary is taller than at least two boys are"

M-theory: Mary's height $\in\{$ d: exactly two boys are not d-tall $\}$

(38) Mary is taller than 10 to15 boys are.

\#E-theory: $\{\mathrm{d}$ : Mary is d-tall $\} \cap\{\mathrm{d}: 10$ to 15 boys are not d-tall $\} \neq \varnothing$ [weak]

"Mary is taller than at least ten boys are"

M-theory: Mary's height $\in\{\mathrm{d}: 10$ to 15 are not d-tall $\}$

Interestingly, in these cases, we see the comparative apparently imposing a maximum on the measure of the subject. For example, (37) says that Mary cannot be taller than the third shortest boy, if there is one. E-theory is not capable of imposing such maximums. This was a plus for E-theory in the DE-Q cases. 
Notice that NM-Qs like exactly two boys can be viewed as the coordination of a UE-Q and a DE-Q: at least two boys and at most two boys. Thus, M-theory's success with exactly two is tied directly to its failure with DEQs. As observed above, DE-Qs in CC are unacceptable, but M-theory predicts that the truth conditions of a comparative sentence with a DE-Q in CC impose a maximum on the measure of the subject. When an NM-Q is in CC, the truth conditions impose both a lower bound and a maximum on the measure of the subject. The UE-Q part of exactly two is responsible for the lower bound; the DEQ part is responsible for the maximum. So, M-theory succeeds with NM-Qs for the very reason it fails with DE-Qs. Now we turn to E-theory's problems with NM-Qs.

\subsection{E-theory on NM quantifiers}

We have seen that E-theory can capture unacceptability of DE-Qs. However, Etheory fails with NM-Qs, predicting truth conditions that are too weak. For example, E-theory currently predicts that (39) is true if and only if Mary is taller than at least two boys. Note that E-theory does not impose a maximum on the measure of the subject when $\mathrm{CC}$ contains something $\mathrm{DE}$; in the NM case, a maximum is required. In this section, I address the question of how E-theory can be fixed on this point.

(39) Mary is taller than exactly two boys are.

\#E-theory: $\{\mathrm{d}$ :Mary is $\mathrm{d}$-tall $\} \cap\{\mathrm{d}$ :exactly 2 boys are not $\mathrm{d}$-tall $\} \neq \varnothing$ [weak]

Our goals for E-theory are (i) to rule out the weak reading that E-theory generates for NM-Qs and (ii) to derive the correct readings, that is, to find a way to impose maximums on the measure of the subject consistent with E-theory. I propose that goal (i) can be accomplished through a plausible pragmatic economy condition and that goal (ii) follows from the proper analysis of the non-monotonic expressions in question.

First notice that, in practice, UE-Qs in CC can impose maximums on the measure of the subject by scalar implicature.

(40) Mary is taller than some of the boys are.

Implicature: Mary is not taller than all the boys are.

(41) Bill is taller than two students are.

Implicature: Bill is not taller than three students are.

When I assert that Bill is taller than two students are, I implicate that I do not have evidence that Bill is taller than three students are. If we add in the assumption that I am informed, we can infer that I know that he is not taller than three. No matter our theory of the comparative, we can derive this as a global implicature when CC contains a weak scalar UE-Q. 
I suggest that we use this as our model for how to derive maximums in the truth conditions of comparative sentences containing NM-Qs. Landman (1998) and Krifka (1999) draw our attention to important parallels between the negative implications of NM-Qs like exactly two and the products of implicaturegenerating mechanisms. Landman observes that analyzing exactly two $N P$ as a semantic unit will not derive the correct truth conditions for the cumulative reading of (42), under which (42) is true iff exactly two students danced with professors and exactly two professors danced with students.

(42) Exactly two students danced with exactly two professors.

(43) Two students danced with two professors.

Landman suggests that semantically exactly two means the same as two but comes with the requirement that it be strengthened by implicature-generating mechanisms. In other words, the at most two component of exactly two's meaning comes from the same place as two's scalar implicatures. Hence, for Landman, the truth conditions of (42) are derived from the truth conditions and implicatures of (43). I refer the reader to Landman (1998) for details.

The implicature-generating mechanism I assume is inspired by Fox's (2007) covert alternative-sensitive EXH, cf. (45). ${ }^{8}$ Incorporating Landman's insights, the Logical Form of exactly two is given in (44). I mark the 'focus' of EXH with bold.

(44) a. Exactly two students smoke.

b. Logical Form: EXH[ 2 students smoke]

$$
\operatorname{EXH}\left(\mathrm{p}_{\mathrm{st}}\right)\left(\mathrm{A}_{<\mathrm{st}, \mathrm{t}}\right)(\mathrm{w}) \text { iff } \mathrm{p}(\mathrm{w})=1 \& \forall \mathrm{q} \in \mathrm{A}[\mathrm{q}(\mathrm{w})=1 \rightarrow \mathrm{p} \Rightarrow \mathrm{q}]
$$

Generally, the EXH triggered by exactly takes local scope. Observe, for example that a weaker wide-scope reading for EXH is unavailable in (46).

(46) Every boy read exactly two books

a. Every boy ${ }_{\mathrm{x}} \mathrm{EXH}[\mathrm{x}$ read $\underline{\mathbf{2}}$ books]

b. \#EXH[every boy read $\underline{\mathbf{2}}$ books] (unavailable meaning)

"Every boy read two books and not every boy read three books"

Under E-theory, as we have already observed, when EXH takes narrow scope that is within $\mathrm{CC}$, the result is truth conditions that are too weak.

\section{Local Scope EXH}

Bill is taller than exactly two students are.

$\# \exists \mathrm{d}[$ Bill is d-tall and $\mathrm{EXH}[\underline{\mathbf{2}}$ students are not d-tall]] (wrong meaning)

${ }^{8}$ Fox (2007) develops a more sophisticated method of picking out the alternatives to be negated. Such developments are unnecessary for the simple cases considered here. 
"Bill is taller than (at least) two students are."

Notice, in particular, that under E-theory, the narrow scope LF is predicted to have the same truth conditions as a sentence in which there is no occurrence of $\mathrm{EXH}$.

I propose to rule out this reading by means of a pragmatic principle that governs the use of EXH. Specifically, I propose that when EXH is triggered, as by exactly, it must have an effect on truth conditions. In (47), it has no effect: (48a) and (48b) are equivalent.

a. $\exists$ d[Bill is d-tall and EXH[ $\underline{\mathbf{2}}$ students are not d-tall]]

b. $\exists \mathrm{d}[$ Bill is $\mathrm{d}$-tall and 2 students are not $\mathrm{d}$-tall]

I must be pointed out that the equivalence between (48a) and (48b) holds only under the assumption that the $\mathrm{CC}\{\mathrm{d}: \mathrm{EXH}[\underline{\mathbf{2}}$ students are not d-tall] $\}$ is nonempty, which I have already suggested is presupposed. Otherwise, the LFs would have different truth conditions. In particular, if there is a tie for second shortest student, then there would be no degrees to which exactly two students are tall, but there would still be a non-empty set of degrees to which (at least) two students are tall. In such a situation, (48b) could be true while (48a) is false. As long as we assume that $\mathrm{CC}$ is presupposed to be non-empty, we see that (48a) and (48b) have the same truth value whenever they are both defined.

Furthermore, I propose that when local scope yields no change in truth conditions, EXH is permitted to apply with wider scope. For example, due to the equivalence of (48a) and (48b), EXH is allowed to take widest scope in the LF of (47).

\section{Wide Scope EXH}

Bill is taller than exactly two students are.

$\mathrm{EXH}[\exists \mathrm{d}[$ Bill is d-tall and $\underline{\mathbf{2}}$ students are not d-tall]]

This LF has distinct truth conditions from the LF with no EXH cf. (48). This LF, unlike (48), says that Bill cannot be taller than the third shortest student, as desired. Hence, the maximum on the measure of the subject derives not from the semantics of the comparative but from the grammatically triggered 'implicature' of the quantifier contained in the than-clause.

To summarize, E-theory faces prima facie difficulties with non-monotonic quantifiers. Specifically, it predicts weak truth conditions, failing to impose necessary maximums on the measure of the subject. I have proposed that this is not a problem for E-theory. The maximums derive not directly from the interpretation of the comparative but from the interpretation of the NM-Qs. Following Landman (1998) and Krifka (1999), I assume that the DE component of quantifiers like exactly two derives from the implicature-generating mechanism, EXH. The insertion of EXH, I propose, is subject to an economy condition requiring it to have an effect on truth conditions. When EXH fails to have an effect locally, it can apply at a higher position in the LF. In the case of 
exactly this derives the maximum on the measure of the subject. In the next section, we examine some other cases of non-monotonic quantifiers.

Beck $(2008 \mathrm{a} \& \mathrm{~b})$. arrives at a similar conclusion about exactly for related but different reasons in an interval-based semantics for comparatives. Also see Landman (2005) for a different approach to this problem.

\subsection{Other Non-Monotonic Quantifiers}

In this section, we will consider to what extent the analysis of exactly applies to other cases of NM-Qs. We examine two cases: continuous and non-continuous non-monotonic quantifiers. Continuity for quantifiers is defined as in (50), cf. Keenan (1996).

$$
\mathrm{Q} \text { is continuous iff for all } \mathrm{X}, \mathrm{Y}, \mathrm{Z} \text { if } \mathrm{X} \in \mathrm{Q} \& \mathrm{Z} \in \mathrm{Q} \& \mathrm{X} \subseteq \mathrm{Y} \subseteq \mathrm{Z} \text {, then } \mathrm{Y} \in \mathrm{Q}
$$

Exactly $n \mathrm{NP}$ is a continuous NM-Q. I will show below that other continuous NM-Qs, such as between $n$ and $m$ NP can fall under the same analysis in comparatives as exactly. Non-continuous quantifiers, like an even number of NPs, on the other hand present something of a problem.

First, consider the continuous NM-Q 10 to 15 . For (51) to be true, Mary must be taller than 10 but not taller than 16 boys. How could we derive such a meaning in the manner we used for exactly.

Mary is taller than 10 to 15 boys are.

I propose that the expression 10 to 15 is an indefinite ranging over numbers between 10 and 15 and that at LF it sits in the degree argument slot of a covert version of Hackl's (2000) many. ${ }^{9}$

$$
\llbracket \text { many } \rrbracket=\lambda d \cdot \lambda P . \lambda Q \cdot|P \cap Q| \geq d
$$

The objects quantified over by the indefinite 10 to 15 are ordered. Given such a semantics, in positive environments, quantifying over the closed interval $[10,15]$ is the same as just using 10. So, an EXH must apply within the scope of the indefinite 10 to 15 in order for the quantification over numbers to make sense.

n to $m$ NPs VP

logical form: $\exists \mathrm{d} \in[\mathrm{n}, \mathrm{m}] \mathrm{EXH}[\underline{\mathbf{d}}$-many NPs VP ]

Notice that when both the indefinite over numbers and EXH scope within CC, there is no effect on the truth conditions under E-theory. The set of degrees (54a) is always an initial segment of (54b) (when both are defined). If the subject's set of degrees overlaps one, it overlaps the other.

${ }^{9}(52)$ is a simplification of Hackl's proposal, ignoring plurality. 
a. $\{\mathrm{d}: \exists \mathrm{n} \in[10,15] \mathrm{EXH}[\underline{\mathbf{n}}$-many boys are not d-tall $]\}$

b. $\{d: \exists n \in[10,15][n-m a n y$ boys are not d-tall $]\}$

This licenses wide scope for EXH. The indefinite quantifier over numbers must then also scope out, perhaps by choice function. ${ }^{10}$ This gives us (55) as the final logical form for (51).

$\exists \mathrm{n} \in[10,15][\mathrm{EXH}[\exists \mathrm{d}[$ Mary is d-tall and $\underline{\mathbf{n}}$-many boys are not d-tall]]]

Essentially, this LF says that there is a number $\mathrm{n}$ between 10 and 15 such that Mary is taller than exactly $n$ boys. Crucially, this entails that she must be taller than 10 boys and can't be taller than 16 .

We encounter a hiccup for E-theory when we look at non-continuous quantifiers like an even number of NPs. The reason is that we cannot force EXH to scope out of CC because it has no effect on truth conditions. EXH does have an effect on truth conditions. I assume the analysis in (57) for an even number.

(56) Mary is taller than an even number of boys are.

\section{an even number of NPs VP}

logical form: $\exists \mathrm{n} \in\{\mathrm{m}: \mathrm{m}$ is even $\}[\mathrm{EXH}[\underline{\mathbf{n}}-\mathrm{many} \mathrm{NPs} \mathrm{VP}]]$

Even when both are non-empty, it will not always be the case that (58a) and (58b) share the same lower bound. Hence, adding EXH within CC does have an effect under E-theory.

(58) a. $\{\mathrm{d}: \exists \mathrm{n} \in\{\mathrm{m}: \mathrm{m}$ is even $\} \mathrm{EXH}[\underline{\mathbf{n}}$-many boys are not $\mathrm{d}$-tall $]\}$

b. $\{\mathrm{d}: \exists \mathrm{n} \in\{\mathrm{m}: \mathrm{m}$ is even $\}$ [n-many boys are not $\mathrm{d}$-tall] $\}$

Consider this scenario: There are 4 boys. One shorter than 5 '. Two are exactly $5^{\prime}$. One is $5{ }^{\prime} 2^{\prime \prime}$. In this scenario, both sets are non-empty, but $5^{\prime} 1^{\prime \prime} \in(58 \mathrm{~b})$ and 5,1 ' $\notin(58 a)$.

As it stands then, E-theory predicts that (56) could be true in a scenario in which Mary is taller than exactly five boys, contrary to fact. At this point, I do not have a solution to this problem. One possibility might be to restrict the models in which we assess the contribution of EXH to those in which measure function are injective, i.e., all individuals are distinguished from each other. Presently, I have no motivation for making such a restriction.

\footnotetext{
${ }^{10} \mathrm{I}$ assume that EXH cannot take an operator's $\mathrm{O}$ trace as focus when $\mathrm{O}$ is within EXH's scope, as this leads to vacuous quantification in the focus alternatives.
} 


\section{Additional Evidence in Favor of E-theory}

M-theory treats all non-monotonic DPs alike and is successful with exactly n, between $n$ and $m$ and an even number. E-theory on the other hand depends on the scope of EXH for its successful analysis of exactly $n$ and between $n$ and $m$ and faces a challenge in an even number. Landman (1998) and Krifka (1999) provide compelling arguments for the analysis of exactly $n$ into a numeral and an implicature mechanism - EXH for us. But not all non-monotonic DPs lend themselves to this kind of an analysis. A non-monotonic DP whose structure does not involve EXH would help us to decide between E-theory and M-theory. I think that explicit coordinations of UE- and DE- Qs are such DPs.

The explicit coordination of a UE-Q and a DE-Q does not derive its maximum from EXH, but from the DE-Q. M-theory predicts such a coordination to impose a maximum on the measure of the subject.

(59) a. *Bill is taller than some girls but no boys are.

b. ${ }^{*}$ Bill is taller than every boy but not every girl is.

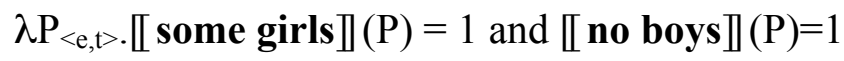

These sentences not only fail to impose maximums, they are simply unacceptable.

(61) *Bill is taller than some girls but no boys are.

E-theory: $\exists \mathrm{d}[$ Bill is d-tall \& some girls but no boys are not d-tall]

Bill is taller than some girls are.

E-theory, on the other hand, predicts that the presence of the DE conjunct will have no effect on truth conditions. ${ }^{11}$ That is, E-theory predicts (61) to be equivalent to (62). As an extension of our earlier principle, I suggest this triviality of contribution as the source of unacceptablity.

Conjunctions of determiners are predicted to have the same status.

(63) ??Bill is taller than some but not all the students are.

??Bill is taller than more than 2 but fewer than 7 students are.

Judgments on these vary; but for nearly all my informants they are an improvement over (59). I do not know why this is so.

\section{Conclusion}

E-theory of the semantics of comparatives (6) provides a better account of the interpretation of the full range of DP quantifiers in comparative clauses than Mtheory (4). M-theory accounts for the interpretation of UE and NM quantifiers,

\footnotetext{
${ }^{11}$ Again, under the presupposition that $\mathrm{CC}$ is non-empty.
} 
but does not provide a principled approach to DE quantifiers. E-theory, when supplemented with the correct approach to NM-Qs, accounts for UE, DE and NM quantifiers - though a problem of over-generation remains for E-theory with noncontinuous NM-Qs.

This paper has specifically addressed the interpretation of DP quantifiers in comparative clauses. These quantifiers are marked out as a class due to the Heim/Kennedy Generalization, see (64) below. Reconciling these results with an approach to non-DP-quantifiers is a goal for further research. Also, the use of negation in the than-clause must be refined to accommodate differentials.

\section{Appendix: Logical Form}

I assume a Bresnan 1973-style syntax: Bill is taller than every girl is

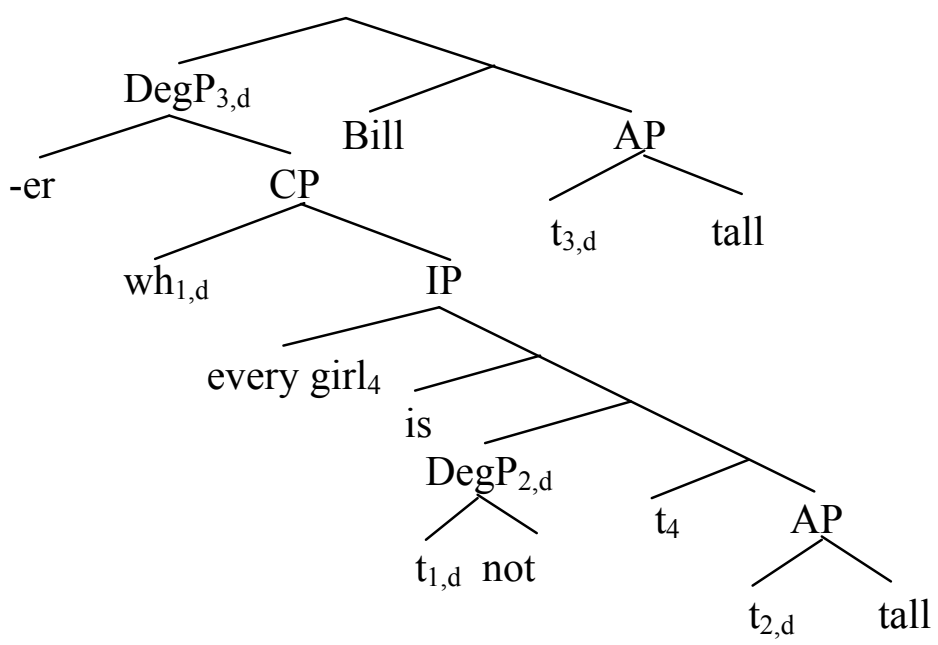

Degree negation: $\quad \llbracket$ not $\rrbracket=\lambda \mathrm{d} . \lambda \mathrm{f}_{<\mathrm{d}, \mathrm{t} .} \mathrm{f}(\mathrm{d})=0 \quad$ see Heim's (2006b) little

M-theory: $\quad$ I -er $\mathbb{\|}=\lambda \mathrm{P}_{<\mathrm{d}, \mathrm{t},} \cdot \lambda \mathrm{Q}_{<\mathrm{d}, \mathrm{t}>} . \max (\mathrm{Q}) \in \mathrm{P}$

E-theory: $\quad \llbracket$-er $\mathbb{\|}=\lambda \mathrm{P}_{<\mathrm{d}, \mathrm{t}}: \mathrm{P} \neq \varnothing . \lambda \mathrm{Q}_{<\mathrm{d}, \mathrm{t}} \triangleright \mathrm{P} \cap \mathrm{Q} \neq \varnothing$

The DegP embedded in the than-clause is capable of taking higher scope. This is apparently necessary for certain modals: allow, have to, require etc. For DPs it is not possible:

(64) Heim/Kennedy Generalization

If the scope of a quantificational DP contains the trace of a DegP, it also contains that DegP itself.

(Heim 2000)

\section{References}

Barwise, Jon and Robin Cooper: 1981, 'Generalized Quantifiers and Natural 
Language', Linguistics and Philosophy 4, 159-219.

Beck, Sigrid: 2008a, 'Comparatives and Superlatives', (eds.) K. von Heusinger, C. Maienborn \& P. Portner. Handbook of Semantics (to appear).

Beck, Sigrid: 2008b, Quantifiers in than-clauses, Ms. Tübingen

Bresnan, Joan: 1973, 'Syntax of the Comparative Clause Construction in English', Linguistic Inquiry 4, 275-343.

Chierchia, Gennaro: 1984, Topics in the Syntax and Semantics of Infinitives and Gerunds, Doctoral Dissertation, UMass, Amherst.

Von Fintel, Kai: 1993, 'Exceptive Constructions', Natural Language Semantics 1, 123-148

Fox, Danny: 2007, 'Free Choice Disjunction and the Theory of Scalar Implicatures', in U. Sauerland \& P. Stateva (eds.) Presupposition and Implicature in Compositional Semantics, Palgrave MacMillan, New York.

Fox, Danny and Martin Hackl: 2006, 'Universal Density of Measurement', Linguistics and Philosophy 29, 537-586.

Gajewski, Jon: 2002, 'L-analyticity and Natural Language', Ms. MIT.

Heim, Irene: 2000, 'Degree Operators and Scope', in B. Jackson and T. Matthews (eds.) Proceedings of SALT X, CLC Publications, Cornell University, Ithaca.

Heim, Irene: 2006a, 'Remarks on Comparative Clauses as Generalized Quantifiers', Ms. MIT.

Heim, Irene: 2006b, 'Little', in M. Gibson and J. Howell (eds.) Proceedings of SALT XVI. CLC Publications, Cornell University, Ithaca.

Kennedy, Christopher: 1999, Projecting the Adjective: the Syntax and Semantics of Gradability and Comparison, Garland, New York.

Krifka, Manfred. 1999. 'At Least Some Determiners aren't', in K. Turner (ed.) The Semantics/Pragmatics Interface from Different Points of View, Elsevier.

Ladusaw, William. A.: 1986, 'Principles of Semantic Filtering', in M. Dalrymple et al. (eds.) Proceedings of WCCFL 5.

Landman, Fred: 1998, Plurals and Maximalization, in S. Rothstein (ed.) Events and Grammar, Kluwer.

Landman, Fred: 2005, 'The Almost (but not Quite) Naïve Theory of Measures', Ms. Tel Aviv University.

Rullmann, Hotze: 1995, Maximality in the Semantics of Wh-Constructions. Doctoral Dissertation, UMass Amherst.

Schwarzschild, Roger and Karina Wilkinson: 2002, 'Quantifiers in Comparatives: a Semantics of Degree Based on Intervals', Natural Language Semantics 10,1-41.

Schwarzschild, Roger: 2004, 'Scope-splitting in the Comparative', Ms. Rutgers.

Schwarzschild, Roger: 2008, 'The Semantics of Comparatives and Other Degree Constructions', Language and Linguistics Compass 2,308-331.

Seuren, P. A. M.: 1973, 'The Comparative', in F. Kiefer and N. Ruwet (eds) Generative Grammar in Europe, Riedel, Dordrecht.

von Stechow, Arnim: 1984, 'Comparing Semantic Theories of Comparison', Journal of Semantics 3, 1-77. 